\title{
EFEITO DO TRATAMENTO TÉRMICO SOBRE O COEFICIENTE DE ENCRUAMENTO DE UMA LIGA AI-Cu-Fe-Zr*
}

\section{Resumo}

Marcos Felipe Macêdo Cardoso ${ }^{1}$ Anna Márcia Rocha Rodrigues ${ }^{2}$ Carlos Alexandre Cavalcante de Sousa ${ }^{3}$ Emerson Rodrigues Prazeres ${ }^{4}$ Everaldo Afonso Fernandes ${ }^{5}$ José Maria do Vale Quaresma ${ }^{6}$

Com o aumento dos investimentos energéticos na região amazônica, surge a necessidade de recapacitação das linhas de transmissão existentes, a fim de mitigar as interrupções de fornecimento de energia para os grandes centros urbanos. Dentro deste contexto, as ligas termorresistentes possuem alta capacidade de transmissão de eletricidade, propondo-se avaliar a influência do tratamento térmico sobre as propriedades da liga Al-0,05\% Cu-[0,35-0,45]\%Fe modificada pelo teor de $0,15 \% Z r$. Os lingotes foram obtidos por fundição direta em coquilha metálica, usinados e envelhecidos artificialmente por 04 (quatro) horas a $150^{\circ} \mathrm{C}$ e comparado seus resultados para a mesma liga sem tratamento. A influência do tratamento térmico foi avaliada através da caracterização macroestrutural, elétrica (ensaio de resistividade) e mecânica (ensaio de tração). Foi selecionado o diâmetro que apresentou os melhores resultados nos ensaios, para realização dos testes de termorresistividade, segundo o Protocolo COPEL e a Norma ASTM B941-10. O tratamento térmico provocou ligeiro aumento nos valores de condutividade elétrica e limite de resistência à tração (LRT), sem alteração da macroestrutura. Como destaque, o diâmetro $3,0 \mathrm{~mm}$ apresentou os melhores resultados de LRT e condutividade, cujos fios foram caracterizados como termorresistentes com e sem tratamento térmico segundo o Protocolo Copel, e somente os fios com tratamento térmico segundo a norma ASTM B941-10.

Palavras-chave:Liga Al-Zr; Tratamento térmico; Propriedades elétricas e mecânicas; Coeficiente de encruamento.

\section{STUDY OF THE EFFECT OF HEAT TREATMENT ON THE HARDENING COEFFICIENT OF Al-0,05\% Cu- $[0,35-0,45] \%$ Fe - 0,15\% Zr ALLOY}

\section{Abstract}

With raising of energy investments in Amazonia, the need for reinforce the existing transmission lines increases to mitigate disruptions of energy supply for large urban centers. Within this context, the heat-resistant alloys have high capacity for electricity transmission, proposing to evaluate the influence of heat treatment on the properties of $\mathrm{Al}-0.05 \% \mathrm{Cu}-[0.35-0.45] \% \mathrm{Fe}$ base-alloy modified by the content of $0,15 \% \mathrm{Zr}$. The ingots were obtained by die casting, machined and artificially aged for 4 hours at $150^{\circ} \mathrm{C}$ and compared their results to the same alloy without treatment. The influence of heat treatment was measured by electrical (resistivity test) and mechanical (tensile test) characterization and metallographic analysis of macrostructure. It was selected the wire with the best results in the tests to perform the thermo-resistivity tests, according to COPEL Protocol and ASTM B941-10. The heat treatment caused a slight increase in the values of electrical conductivity and ultimate tensile strength (UTS), without changings in the macrostructure. As a highlight, the 3,0 $\mathrm{mm}$ wires showed the best results of UTS and electrical conductivity, which were characterized 
as thermo-resistant with and without heat treatment according to the Copel Protocol, and only the heat-treated wires according to ASTM B941-10.

Keywords: Al-Zr Alloy; Heat treatment; Electrical and mechanical properties; Strainhardening coefficient.

1 Engenharia Mecânica, Bacharel, Universidade Federal do Pará, Belém, Pará, Brasil.

2 Engenharia Industrial, Me., Programa de Pós-Graduação em Engenharia Industrial, Universidade Federal do Pará, Belém, Pará.

3 Engenharia Mecânica, Me., Universidade Federal do Pará, Belém, Pará, Brasil.

4 Engenharia Mecânica, Me., Doutorando em Engenharia de Recursos Naturais, Programa de PósGraduação em Engenharia de Recursos Naturais da Amazônia, Universidade Federal do Pará, Belém, Pará.

5 Engenharia Mecânica, Me., Professor EBTT, Instituto Federal do Pará, Marabá, Pará.

6 Engenharia Mecânica, Dr., Professor Titular, Faculdade de Engenharia Mecânica, Universidade Federal do Pará, Belém, Pará. 


\section{INTRODUÇÃO}

Existe uma enorme gama de atividades em novas tecnologias para enfrentar a necessidade crescente de energia elétrica. Destacam-se os estudos para o desenvolvimento de materiais que possam suprir com maior eficiência as condições de transporte de energia elétrica, principalmente quando se fala em temperatura [1].

Nas últimas décadas vem crescendo o interesse pelos materiais não-ferrosos, como condutores elétricos, entre os principais utilizados estão o Alumínio (Al) e ligas de $\mathrm{Al}$, que ganham cada vez mais espaço no meio acadêmico devido suas propriedades vantajosas.

Os principais motivos para o consumo tão significativo desse metal são as suas excelentes propriedades físico-químicas, entre as quais se destacam: (a) sua densidade de $2,7 \mathrm{~g} / \mathrm{cm}^{3}$, aproximadamente $1 / 3$ da do aço, o que, somado à sua elevada resistência mecânica, o torna bastante útil na construção de estruturas móveis, como veículos e aeronaves; (b) possui boa moldabilidade, o que garante alta maleabilidade e ductilidade, permitindo à indústria utilizá-lo de diversas formas, pois suas propriedades mecânicas facilitam sua conformação e possibilitam a construção de formas adequadas aos mais variados projetos; (c) o alumínio com determinados tratamentos e/ou elementos de liga torna-se um material com boa resistência mecânica; (d) elevada condutividade elétrica, cerca de $62 \%$ da do cobre e (e) facilidade pela qual o alumínio pode ser fabricado em várias formas é uma das suas mais importantes vantagens. Por causa dessas, e outras vantagens, que o alumínio se tornou um dos metais não ferrosos mais utilizados no mundo.

A aplicação de tratamentos térmicos em material metálico pode provocar alterações significativas nas propriedades do material devido a alterações ocorridas em sua estrutura cristalina [2].

Dentro desse contexto $O$ Grupo de Pesquisa em Engenharia de Materiais GPEMAT/FEM/ITEC/UFPA - têm estudado o melhoramento das ligas de alumínio utilizadas para produção de cabos para transmissão e distribuição de energia elétrica.

Estudos mostram que as propriedades desses cabos condutores, são influenciadas por parâmetros estruturais, assim como o tipo de processo empregado em sua solidificação e os tratamentos térmicos aplicados no material [3]. O objetivo deste trabalho visa analisar o comportamento das propriedades da liga $\mathrm{Al}-0,05 \% \mathrm{Cu}[0,35-$ $0,45] \%-0,15 \% \mathrm{Zr}$ após tratamento térmico de $150^{\circ} \mathrm{C} / 4 \mathrm{~h}$ buscando o conjunto de propriedades mais vantajosas. Desta maneira, busca-se um fio com elevado desempenho eletro condutor e resistência mecânica (LRT) associada a coeficiente de encruamento $(\eta)$ que identifique cabos com maior altura de segurança e minimize os rompimentos das linhas de transmissão.

\section{MATERIAIS E MÉTODOS}

A liga foi obtida a partir de tarugos de alumínio comercialmente puro após serem adicionadas quantidades de cada elemento de liga escolhido de forma a se obter os teores desejados. Os materiais foram inseridos em cadinho de carbeto de silício, próprio para fundição de materiais não-metálicos $A$ aferição da composição foi feita após o vazamento da liga com a utilização de amostra testemunho obtida durante o processo de fundição da liga. 
Realizou-se o vazamento em coquilha metálica em formato de "U", previamente pintado com a solução de caulim e aquecido a $150^{\circ} \mathrm{C}$ para eliminar qualquer umidade presente no mesmo. O material fundido foi desmoldado em amostras com diâmetro de $22 \mathrm{~mm}$, e dividido em dois lotes: no primeiro, foi mantida a condição da estrutura bruta de fusão; o segundo foi submetido a tratamento térmico a $150^{\circ} \mathrm{C} / 4 \mathrm{~h}$. Após, foi realizada a macrografia dos materiais, utilizando-se o reagente "Keller" de composição [ $3 \mathrm{ml} \mathrm{HCl}, 2 \mathrm{ml} \mathrm{HF}, 5 \mathrm{ml} \mathrm{HNO} 3,190 \mathrm{ml} \mathrm{H}_{2} \mathrm{O}$ ]. As amostras foram usinadas para o diâmetro de $18,5 \mathrm{~mm}$ e laminadas para os diâmetros $[4,0 ; 3,8 ; 3,0$ e 2,7] mm, para realização dos ensaios de resistividade elétrica e ensaio de tração.

O ensaio de resistividade elétrica, para obtenção da condutividade elétrica [\%IASC], foi realizado com Micro ohmímetro Megabrás (Ponte de Kelvin) modelo MPK-2000; e o ensaio de tração, para obtenção das propriedades mecânicas, foi realizado em uma máquina de tração Kratos, modelo KE 3000MP.

Inicialmente, foi realizada a análise química da liga a partir da amostra-testemunho, utilizando-se um analisador químico do tipo espectrômetro óptico.

As amostras foram divididas em dois lotes: i. Deformadas sem tratamentos térmico [STT] e ii. Tratadas a $150^{\circ} \mathrm{C} / 4 \mathrm{~h}$, e posteriormente deformadas [TT $150^{\circ} \mathrm{C} / 4 \mathrm{~h}$ ].

A partir dos resultados de [\%IACS] e LRT da liga base STT e TT $150^{\circ} \mathrm{C} / 4 \mathrm{~h}$, foram realizados os estudos e avaliações dos resultados obtidos nos diferentes diâmetros conformados. As amostras foram caracterizadas sequencialmente quanto à condutividade elétrica, quanto ao LRT e quanto à ductilidade. Ressalte-se que a avaliação quanto à ductilidade foi realizada segundo adaptação da norma ASTM E112.

Finalmente para o melhor diâmetro, aplicou-se o teste de termorresistência, de acordo com o protocolo COPEL (Companhia Paranaense de Energia Elétrica) e a Norma ASTM B941-10 [4]; [5] para a constatação da termorresistividade da liga.

\section{RESULTADOS E DISCUSSÃO}

\subsection{Análise química e macroestrutura}

A liga foi preparada utilizando-se cálculos estequiométricos, a fim de se obter os teores desejados dentro das devidas proporções. Após fundição, foi realizada a análise química, cujos resultados obtidos podem ser visualizados na Tabela 1, indicando que todos os procedimentos foram efetuados corretamente e a composição está em concordância com o escopo deste trabalho.

Tabela 1. Composição química para as ligas desejadas e obtidas.

\begin{tabular}{cccc}
\hline Liga & \multicolumn{3}{c}{ Elementos de liga (\%p) } \\
\hline Al $0,05 \mathrm{Cu}-[0,35-0,45] \%$ & $\mathrm{Cu}$ & $\mathbf{F e}$ & $\mathbf{Z r}$ \\
$\mathrm{Fe}-0,15 \% \mathrm{Zr}$ & 0,045 & 0,397 & 0,148 \\
\hline
\end{tabular}

Após a confirmação da análise química, foi procedida a caracterização das macroestruturas da liga para as condições com tratamento térmico (TT $150^{\circ} \mathrm{C} / 4 \mathrm{~h}$ ) e sem tratamento térmico (STT), que estão representadas na Figura 1. 

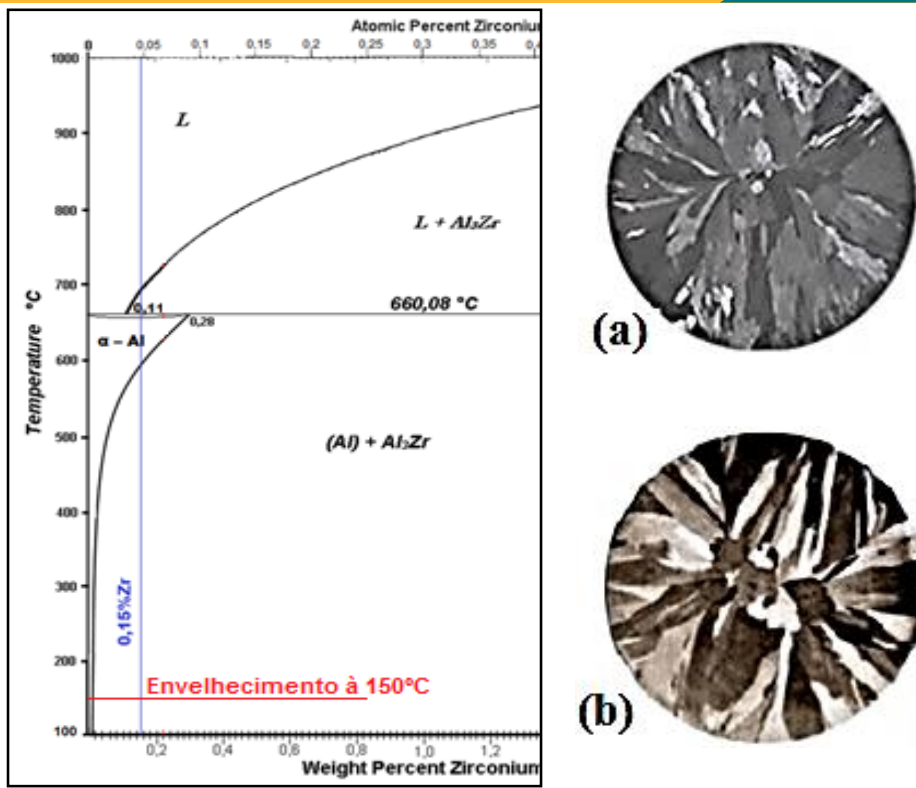

(b)

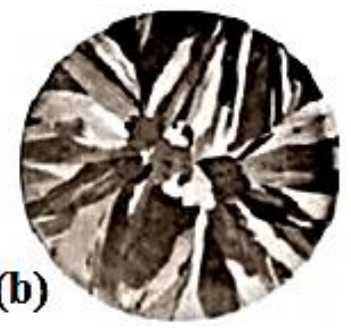

Figura 1. Macroestrutura da liga nas condições (a) STT (b) $\operatorname{TT} 150^{\circ} \mathrm{C} / 4 \mathrm{~h}$.

As macro estruturas das ligas foram reveladas através de ataque químico, utilizando-se reagente "Keller" a 0,5\%. Pode ser observado para a liga STT (a) formato predominante de grãos colunares e, após tratamento térmico, macrografia (b), não houve modificação significativa da estrutura granular. Assim, considera-se que o tratamento térmico nas condições utilizadas não foi suficiente para provocar modificação da macroestrutura.

É conveniente ressaltar que as amostras foram divididas em dois (02) grupos para serem avaliadas:

i. O primeiro grupo foi caracterizado sem tratamento térmico, na condição como fundida que a liga apresenta à temperatura ambiente, 0 arranjo estrutural de solução sólida supersaturada de $\mathrm{Zr}$ em Al, obtido através de resfriamento sob condições moderadas em coquilha metálica, cuja taxa de resfriamento tende a impedir a difusão de $\mathrm{Zr}$, deixando-o retido na matriz devido à têmpera líquida;

ii. O segundo grupo foi caracterizado pós tratamento térmico, em que a baixa temperatura está associada a tempo relativamente longo, caracterizando efetivo procedimento de alivio de tensões.

\subsection{Caracterização Elétrica}

$\mathrm{Na}$ Tabela 2, tem-se listados os resultados da condutividade elétrica para a liga nas duas condições, ilustrados na Figura 2.

Tabela 2. Resultados de Condutividade Elétrica e [ $\eta$ para a liga STT e TT $150^{\circ} \mathrm{C} / 4 \mathrm{~h}$.

\begin{tabular}{|c|c|c|c|c|}
\hline \multirow{2}{*}{ Diâmetro [mm] } & \multicolumn{2}{|c|}{ Condutividade Elétrica [\% IACS] } & \multicolumn{2}{|c|}{ Coeficiente de Encruamento [ $\eta]$} \\
\hline & STT & TT $150^{\circ} \mathrm{C} / 4 \mathrm{~h}$ & STT & TT $150^{\circ} \mathrm{C} / 4 \mathrm{~h}$ \\
\hline 2,7 & 58,72 & 56,04 & 0,82 & 0,58 \\
\hline 3,0 & 59,90 & 57,22 & 0,67 & 0,51 \\
\hline 3,8 & 53,10 & 52,95 & 0,90 & 0,71 \\
\hline 4 & 54,00 & 53,58 & 0,97 & 0,93 \\
\hline
\end{tabular}


A comparação entre as ligas STT e TT $150^{\circ} \mathrm{C} / 4 \mathrm{~h}$ mostra que, após tratamento térmico, houve ligeiras perdas de condutividade elétrica e [ $\eta]$. Dessa forma, o tratamento térmico, ao atuar para o alivio de tensões, provoca a re-acomodação interna do material e, assim, proporciona capacidade de encruamento mais intensa, dificultando a passagem de elétrons em todos os diâmetros.

Os valores obtidos para a condutividade elétrica e para o coeficiente de Encruamento [ $\eta$ ] permitem as seguintes avaliações:

i. A liga na condição $\mathrm{TT} 150^{\circ} \mathrm{C} / 4 \mathrm{~h}$ apresenta menor desempenho elétrico,

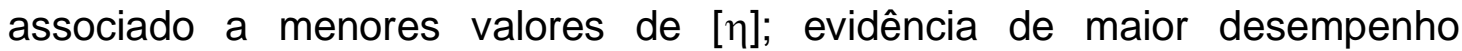
mecânico;

ii. Os fios com menores diâmetros, por isso mais encruado, apresentam maior capacidade de conduzir eletricidade pela manifestação mais intensa do fenômeno da recuperação dinâmica, e estão relacionados aos menores valores de $[\eta]$;

iii. O fio com o diâmetro de 3,0 mm apresenta maiores valores para [\%IACS] associados a menores valores de [ $\eta]$. Neste caso, tem-se maior acumulo de deformação a frio e, como consequência, ocorrência do fenômeno da recuperação dinâmica mais intensa.

Com base nestas observações menores valores para $\circ[\eta]$ pode ser indicativo de material com maior capacidade de conduzir eletricidade.
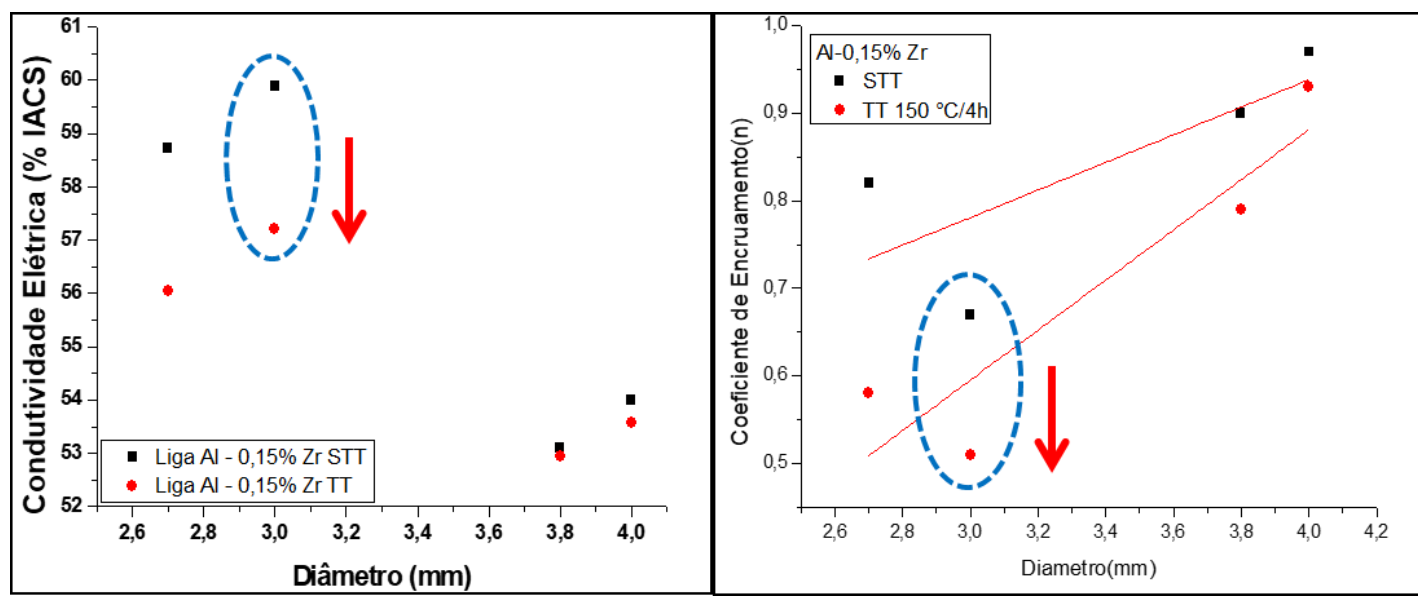

Figura 2. Valores de condutividade elétrica e[ $\eta]$ para a liga base para todos os diâmetros

\subsection{Caracterização Mecânica}

A caracterização mecânica foi realizada a partir da avaliação do LRT, conforme mostrado na Tabela 3 e na Figura 3 , e do coeficiente de encruamento [ $\eta$ p para os todos os diâmetros $[2,7 ; 3,0 ; 3,8 ;$ e 4,0$] \mathrm{mm}$.

Tabela 3. Valores de LRT e do Coeficiente de Encruamento da liga base para todos os diâmetros STT e TT $150^{\circ} \mathrm{C} / 4 \mathrm{~h}$.

\begin{tabular}{|c|c|c|c|c|}
\hline \multirow{2}{*}{ Diâmetro [mm] } & \multicolumn{2}{|c|}{ LRT [MPa] } & \multicolumn{2}{|c|}{ Coeficiente de Encruamento [ $\eta]$} \\
\hline & STT & $\mathrm{TT} 150^{\circ} \mathrm{C} / 4 \mathrm{~h}$ & STT & $\mathrm{TT}^{150^{\circ} \mathrm{C} / 4 \mathrm{~h}}$ \\
\hline 2,7 & 197,52 & 208,67 & 0,82 & 0,58 \\
\hline 3,0 & 204,64 & 214,77 & 0,67 & 0,51 \\
\hline 3,8 & 170,52 & 180,83 & 0,90 & 0,71 \\
\hline 4,0 & 174,24 & 185,36 & 0,97 & 0,93 \\
\hline
\end{tabular}



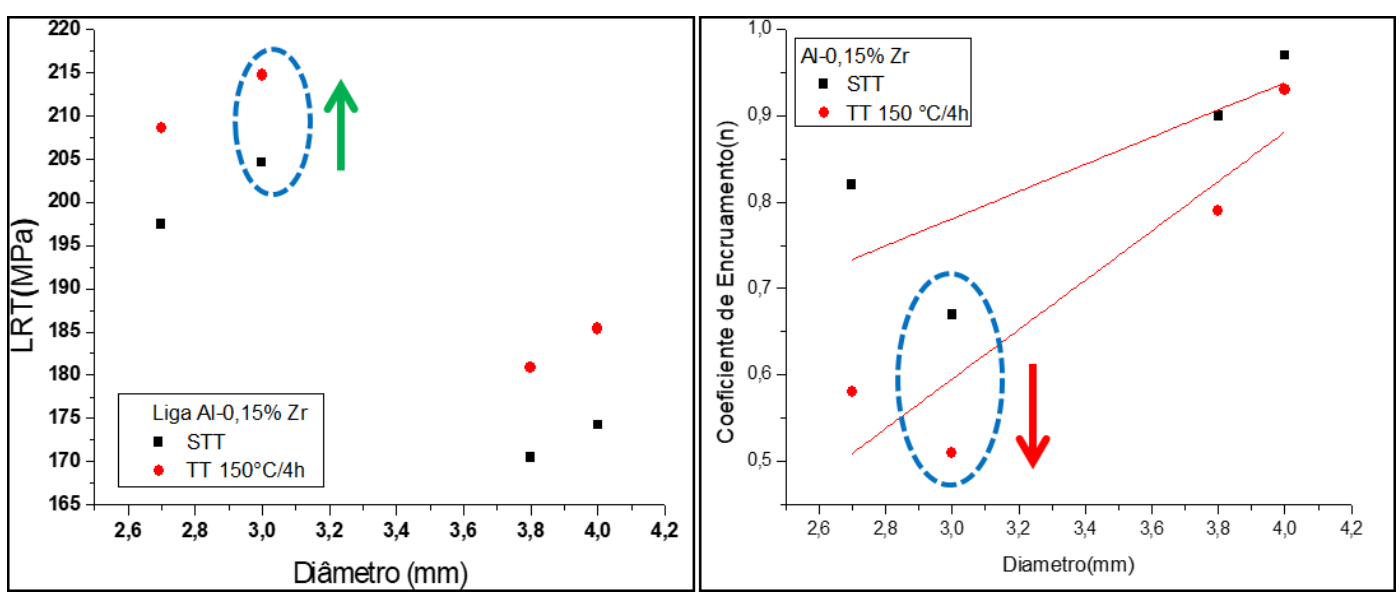

Figura 3. Valores de LRT e o Coeficiente de Encruamento [ $\eta$ da liga base para todos os diâmetros.

Por outro lado, os valores obtidos para o LRT e para o coeficiente de Encruamento

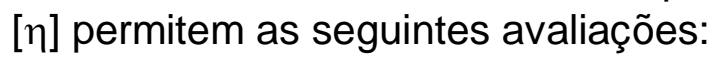

i. Como comentado anteriormente a condição TT $150^{\circ} \mathrm{C} / 4 \mathrm{~h}$ ao atuar para o alivio de tensões, nas estruturas brutas de fusão, proporciona capacidade de encruamento mais intensa que se verifica para todos os diâmetros, como pode ser visto na Figura 3a, e que está associada a menores valores de [ $\eta]$ como pode ser observado na Figura $3 b$;

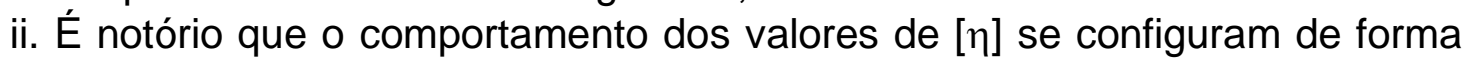
oposta aos valores do LRT como pode ser observado nas figuras 3[a e b].

Baseado nestas duas observações pode ser inferido que maiores valores de [ $\eta$ ] podem ser indicativos de maior ductilidade.

A comparação dos resultados pelos grupos de amostras com e sem tratamento térmico mostra que ocorreram ligeiros ganhos do LRT para as amostras tratadas termicamente. Com isso, a reorganização e a redução das tensões internas que o material possuía devido à têmpera líquida atuaram de forma positiva nos resultados de LRT.

Destaca-se novamente o diâmetro 3,0 $\mathrm{mm}$ por apresentar os maiores valores de LRT, por apresentar maior grau de deformação no processo de conformação plástica, cujo encruamento provocou maior resistência mecânica, seja na condição STT como na condição TT $150^{\circ} \mathrm{C} / 4 \mathrm{~h}$.

\subsection{Avaliação da Ductilidade: Liga STT}

A Tabela 4 e a Figura 4 relacionam os valores de alongamento $(\delta)$, Razão das

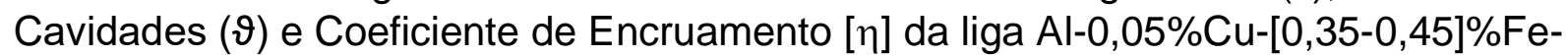
$0,15 \%$ Zr STT em todos os diâmetros.

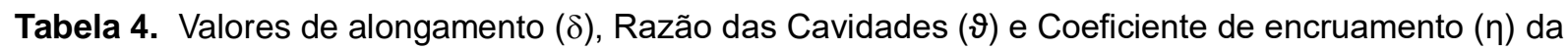
liga base STT para todos os diâmetros.

\begin{tabular}{cccc}
\hline Diâmetro $(\mathbf{m m})$ & {$[\delta](\%)$} & {$[\boldsymbol{\vartheta}]$} & {$[\eta]$} \\
\hline $\mathbf{2 , 7}$ & 4,11 & 1,43 & 0,82 \\
\hline $\mathbf{3 , 0}$ & 3,98 & 1,39 & 0,67 \\
\hline $\mathbf{3 , 8}$ & 5,01 & 1,47 & 0,90 \\
\hline $\mathbf{4 , 0}$ & 5,37 & 1,54 & 0,97 \\
\hline
\end{tabular}



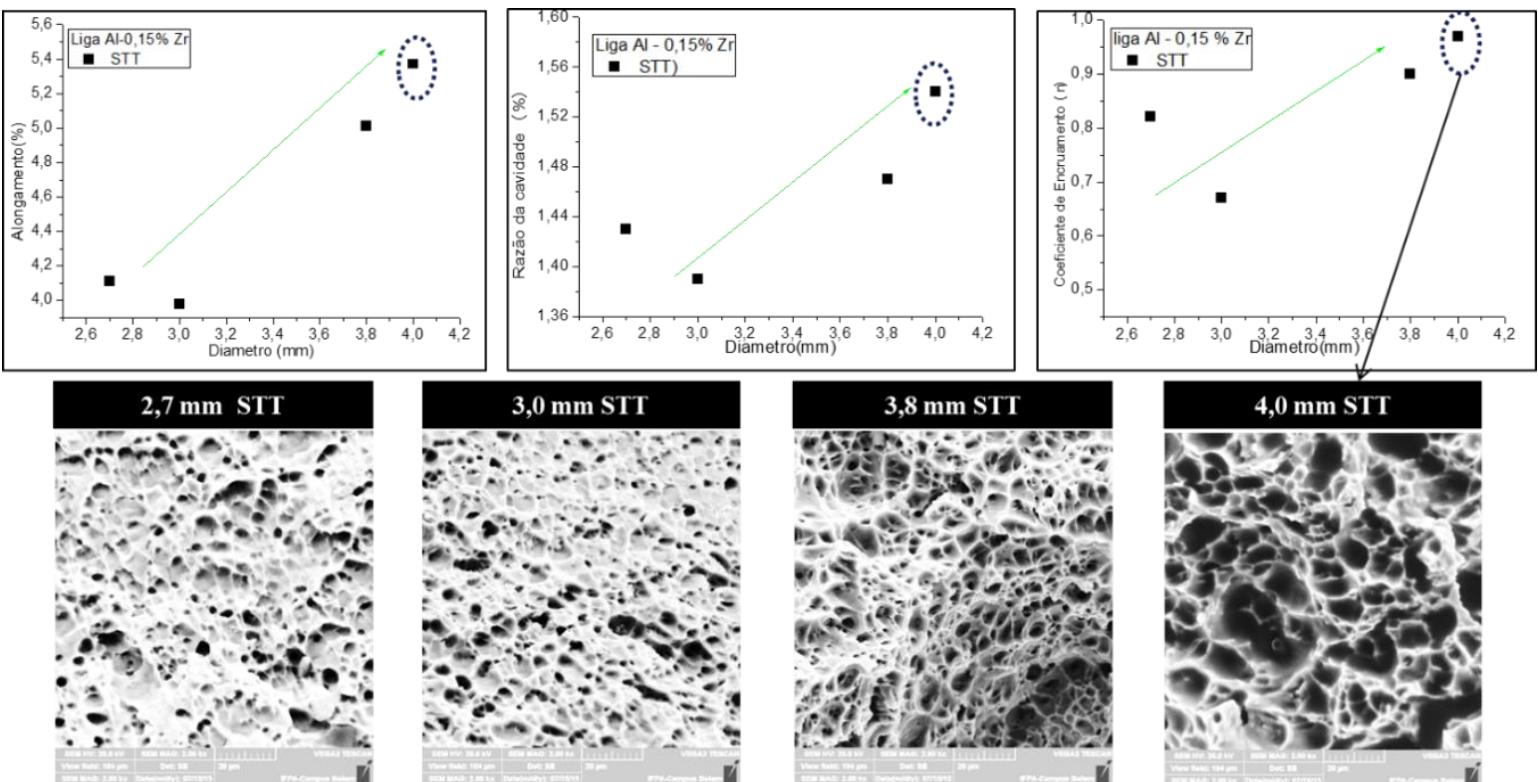

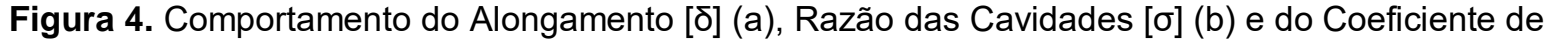
Encruamento [n] (c) e micrografias da topografia da fratura para liga base STT para todos os diâmetros.

\subsection{Avaliação da Ductilidade: Liga TT $150^{\circ} \mathrm{C} / 4 \mathrm{~h}$}

Por outro lado, a Tabela 5 e a Figura 5 relacionam os valores de alongamento $(\delta)$, Razão das Cavidades ( $\vartheta$ e e Coeficiente de Encruamento [ $\eta$ ] da liga Al-0,05\%Cu$[0,35-0,45] \% \mathrm{Fe}-0,15 \% \mathrm{Zr}$ na condição $\mathrm{TT} 150^{\circ} \mathrm{C} / 4 \mathrm{~h}$ em todos os diâmetros.

Tabela 5. Valores de alongamento ( $\delta$ ), Razão das Cavidades $(\vartheta)$ e Coeficiente de encruamento [ $\eta]$ da liga base TT $150^{\circ} \mathrm{C} / 4 \mathrm{~h}$ para todos os diâmetros.

\begin{tabular}{cccc}
\hline Diâmetro $(\mathbf{m m})$ & {$[\delta](\%)$} & {$[\vartheta]$} & {$[\eta]$} \\
\hline $\mathbf{2 , 7}$ & 4,29 & 1,36 & 0,58 \\
\hline $\mathbf{3 , 0}$ & 4,03 & 1,34 & 0,50 \\
\hline $\mathbf{3 , 8}$ & 5,18 & 1,45 & 0,71 \\
\hline $\mathbf{4 , 0}$ & 5,41 & 1,49 & 0,93 \\
\hline
\end{tabular}
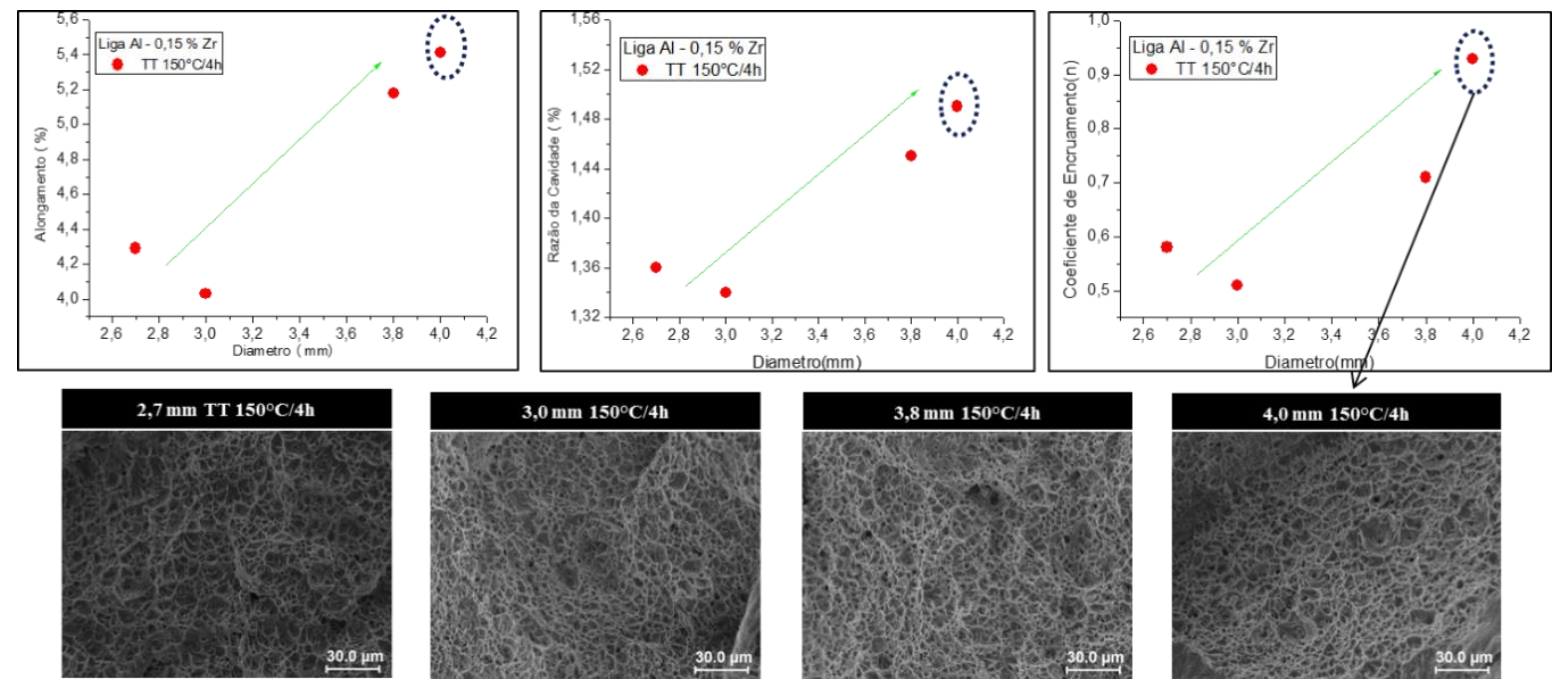

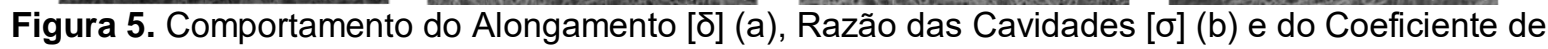
Encruamento [n] (c) TT $150^{\circ} \mathrm{C} / 4 \mathrm{~h}$ para todos os diâmetros. 
No conjunto de informações apresentado nas Figuras 4 e 5, observa-se que, para o comportamento crescentes de alongamento [ $\delta$ ], estão associados a comportamentos

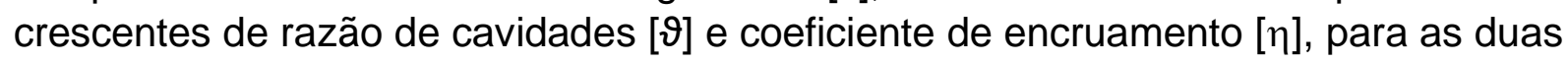
condições em que se encontram as ligas [STT] e $\left[T T 150^{\circ} \mathrm{C} / 4 \mathrm{~h}\right]$. Esse comportamento se reflete nas topografias das fraturas para os diâmetros estudados, como pode ser observado nas fotos micrografias das microcavidades das Figura [4 e 5] e, assim, tem-se microcavidades maiores e mais coalescidas, associadas a maiores valores de $[\delta],[\vartheta]$ e $[\eta]$.

Desta forma é possível inferir-se, para este estudo, que o comportamento crescente

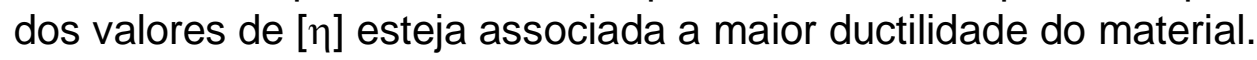

\subsection{Teste de Termorresistividade}

Nessa seção, foi avaliado o comportamento do fio com diâmetro de 3,0 mm quando submetido a dois testes de termorresistividade: Protocolo COPEL e a Norma ASTM 941B-10, que avaliam a termorresistividade do material, característica física muito importante do material quando aplicado na elaboração de linhas de transmissão e distribuição aéreas de energia elétrica.

\subsubsection{TESTE DE TERMORRESISTIVIDADE: $230^{\circ} \mathrm{C} / 1 \mathrm{~h}$ e $280^{\circ} \mathrm{C} / 1 \mathrm{~h}$ PARA LIGA STT}

Os resultados de LRT [MPa] e Porcentagem de perdas de LRT [\%(MPa)] para o teste de termorresistividade da liga sem tratamento térmico prévio (STT), para o diâmetro 3,0 mm, estão relacionados na Tabela 6 e plotados na Figura 6 .

Tabela 6. Teste de Termorresistividade para a liga STT: Valores de (a) LRT [MPa] e Porcentagem de perdas $[\%(\mathrm{MPa})]$ para o diâmetro $3,0 \mathrm{~mm}$.

\begin{tabular}{cccccc}
\hline & STT & \multicolumn{4}{c}{$\mathbf{S T T}_{\mathbf{R}}$} \\
\cline { 2 - 6 } Liga & LRT & LRT & $\begin{array}{c}\text { Perda } \\
\text { (\%) }\end{array}$ & LRT & $\begin{array}{c}\text { Perda } \\
\text { (\%) }\end{array}$ \\
\cline { 2 - 6 } & 204,64 & 202,04 & 1,27 & 169,61 & 17,11 \\
\hline $\begin{array}{c}\text { Al-0,15\%Zr } \\
\text { - STT }\end{array}$ & & & & &
\end{tabular}

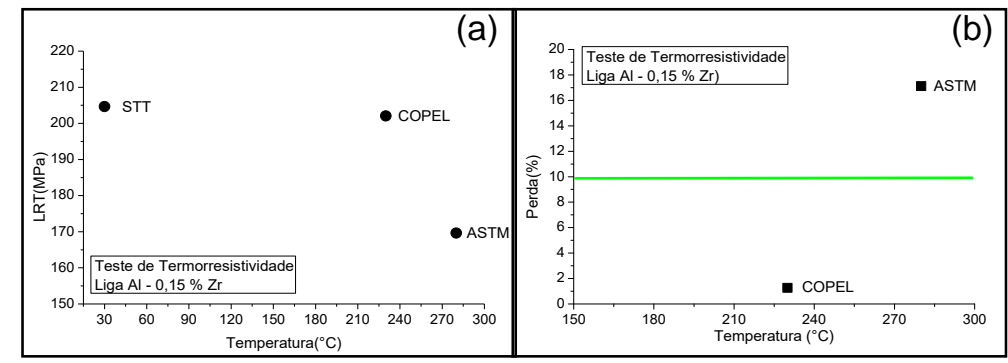

Figura 6. Teste de Termorresistividade a $230^{\circ} \mathrm{C} / 1 \mathrm{~h}$ e $280^{\circ} \mathrm{C} / 1 \mathrm{~h}$ : (a) Valores de LRT; (b) Perdas de LRT da liga STTR, para o diâmetro $3,0 \mathrm{~mm}$.

A análise dos dados permite a constatação de que a liga não se enquadra como termorresistente à temperatura de $280^{\circ} \mathrm{C} / 1 \mathrm{~h}$ por apresentar perdas de $17,12 \%$ do LRT, acima de $10 \%$ admitido pelo teste, como indicado na Figura 6b. É possível que a temperatura utilizada provoque a recristalização, e não a recuperação, como esperada. Diferentemente do resultado à temperatura de $230^{\circ} \mathrm{C} / 1 \mathrm{~h}$, que classifica a liga como termorresistente, como indicado na Figura $6 \mathrm{~b}$. 
3.6.2 TESTE DE TERMORRESISTIVIDADE: $230^{\circ} \mathrm{C} / 1 \mathrm{~h}$ e $280^{\circ} \mathrm{C} / 1 \mathrm{~h}$ PARA A LIGA $\mathrm{TT} 150^{\circ} \mathrm{C} / 4 \mathrm{~h}$

Os resultados de LRT [MPa] e Porcentagem de perdas de LRT [\%(MPa)] para o teste de termorresistividade da liga com tratamento térmico prévio $\left(\mathrm{TT} 150^{\circ} \mathrm{C} / 4 \mathrm{~h}\right)$, para o diâmetro 3,0 mm, estão relacionados na Tabela 7 e plotados na Figura 7.

Tabela 7. Teste de Termorresistividade para a liga TT $150^{\circ} \mathrm{C} / 4 \mathrm{~h}$ : Valores de (a) LRT [MPa] e Porcentagem de perdas [\%(MPa)] para o diâmetro $3,0 \mathrm{~mm}$.

\begin{tabular}{cccccc}
\hline \multirow{2}{*}{ Liga } & STT & \multicolumn{4}{c}{$\mathrm{TT}_{\mathrm{R}} 150^{\circ} \mathrm{C} / 4 \mathrm{~h}$} \\
\cline { 2 - 6 } & LRT & LRT & $\begin{array}{c}\text { Perda } \\
(\%)\end{array}$ & LRT & $\begin{array}{c}\text { Perda } \\
(\%)\end{array}$ \\
\hline $\begin{array}{l}\text { Al-0,15\%Zr - } \\
\text { TT } 150^{\circ} \mathrm{C} / 4 \mathrm{~h}\end{array}$ & 214,77 & 206,37 & 3,91 & 203,73 & 5,14 \\
\hline
\end{tabular}

A análise dos dados permite a constatação de que a liga na condição $T T 150^{\circ} \mathrm{C} / 4 \mathrm{~h}$ se enquadra como termorresistente tanto à temperatura de $230^{\circ} \mathrm{C} / 1 \mathrm{~h}$ quanto a $280^{\circ} \mathrm{C} / 1 \mathrm{~h}$, cujas perdas do LRT foram mantidas abaixo de $10 \%$ admitido pelo teste, como indicado na Figura $7 \mathrm{~b}$. Provavelmente, o tratamento térmico prévio $\left(\mathrm{TT} 150^{\circ} \mathrm{C} / 4 \mathrm{~h}\right)$, ao promover o alívio de tensões da estrutura bruta de fusão e, consequentemente, o rearranjo em uma estrutura com menor concentração de tensões e defeitos, tenha provocado o aumento da temperatura de recristalização, de tal sorte que os tratamentos a $230^{\circ} \mathrm{C} / 1 \mathrm{~h}$ e $280^{\circ} \mathrm{C} / 1 \mathrm{~h}$ tenham sido aplicados na faixa de recuperação da liga.

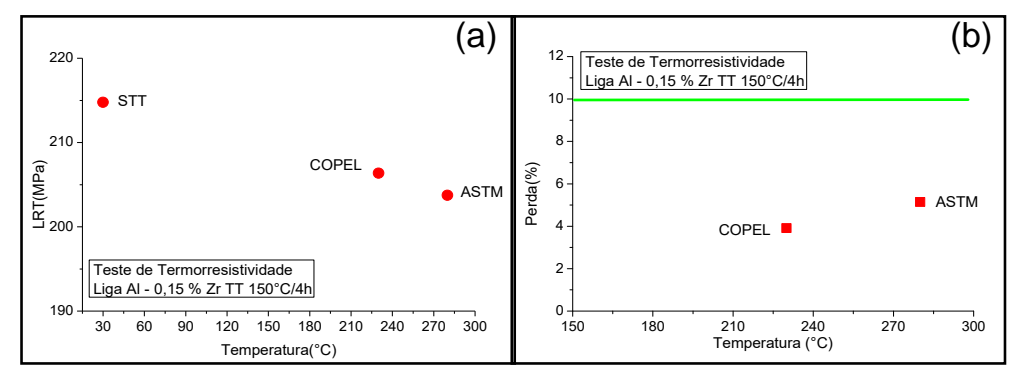

Figura 7. Teste de Termorresistividade a $230^{\circ} \mathrm{C} / 1 \mathrm{~h}$ e $280^{\circ} \mathrm{C} / 1 \mathrm{~h}$ : (a) Valores de LRT; (b) Perdas de LRT da liga $\mathrm{TT}_{\mathrm{R}} 150^{\circ} \mathrm{C} / 4 \mathrm{~h}$, para o diâmetro $3,0 \mathrm{~mm}$.

\section{CONCLUSÕES}

- O tratamento térmico não modificou a estrutura a nível macroestrutural. $\mathrm{E}$, desta forma, provocando apenas alívio de tensões.

- As perdas de $[\eta]$ e condutividade elétrica para a liga com tratamento térmico $\mathrm{TT} 150^{\circ} \mathrm{C} / 4 \mathrm{~h}$ ocorreram devido ao alívio de tensões, que diminuiu a capacidade de recuperação dinâmica da estrutura. Além disso, os menores

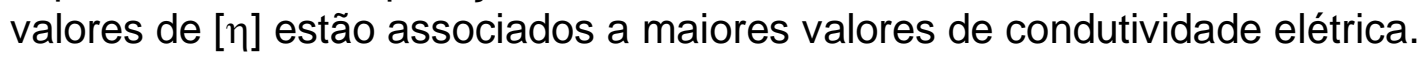

- Os ganhos de LRT e perdas de [ $\eta$ ] para a liga com tratamento térmico $\mathrm{TT} 150^{\circ} \mathrm{C} / 4 \mathrm{~h}$ ocorreram devido à maior capacidade de encruamento da liga.

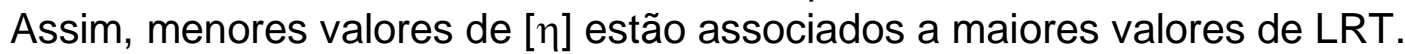

- A maior ductilidade, tanto para a liga na condição [STT] quanto para

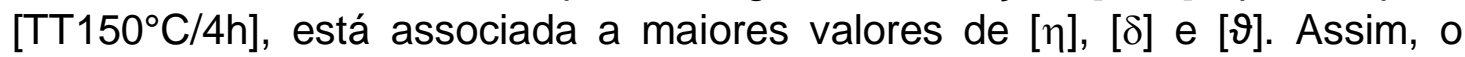
tratamento térmico não interferiu sobre a ductilidade do material. 
- Quanto à termorresistividade a $230^{\circ} \mathrm{C} / 1 \mathrm{~h}$ e $280^{\circ} \mathrm{C} / 1 \mathrm{~h}$, constatou-se que a liga sem tratamento térmico prévio (STT) se enquadrou como termorresistente somente a $230^{\circ} \mathrm{C} / 1 \mathrm{~h}$. Para a liga com tratamento térmico prévio $\left(\mathrm{TT} 150^{\circ} \mathrm{C} / 4 \mathrm{~h}\right)$, constatou-se que a liga se enquadrou como termorresistente para os dois testes de termorresistividade.

\section{Agradecimentos}

Os autores gostariam de agradecer ao Conselho Nacional de Desenvolvimento Científico e Tecnológico (CNPq), CAPES (Coordenação de Aperfeiçoamento de Pessoal de Nível Superior), Universidade Federal do Pará (UFPA) e ITEC (Instituto de Tecnologia/UFPA).

\section{REFERÊNCIAS}

1 ABNT - Associação Brasileira de Normas Técnicas - "Fios e cabos elétricos - Tração à ruptura em componentes metálicos", Rio de Janeiro, Ago. 1981, NBR 6810.

2 ABNT - Associação Brasileira de Normas Técnicas - "Fios de alumínio nus de seção circular para fins elétricos", Rio de Janeiro, 1985, NBR 5118.

3 ABNT - Associação Brasileira de Normas Técnicas - "Fios e cabos elétricos - Ensaio de resistência elétrica", Rio de Janeiro, Mar. 1986, NBR 6814.

4 ABNT - Associação Brasileira de Normas Técnicas - "Fios e cabos elétricos - Ensaio de determinação da resistividade em componentes metálicos", Rio de Janeiro, Ago. 1981, NBR 6815.

5 American Society for Metals (ASM INTERNATIONAL), B941-10. Standard Specification for Heat Resistant Aluminum-Zirconium Alloy Wire for Electrial Purposes - ASTM, 2010.

6 ASKELAND, D. R.; PHULÉ, P. P. Ciência e Engenharia dos Materiais, 1 ed., p. 180. São Paulo, Cengace Learning, 2008.

7 CARDOSO, M. F. M. Estudo do efeito da adição de magnésio sobre propriedades mecânicas, elétricas e estruturais de fios para transmissão e distribuição de energia elétrica da liga Al-0,05\%pCu-[0,24-0,28]\%pFe. [Trabalho de Conclusão de Curso]. Pará. Universidade Federal do Pará; 2013.

8 Companhia Paranaense de Energia - COPEL. Disponível em: http://www.copel.com/hpcopel/root/index.jsp. Acesso em: 09 mai. 2017.

9 DIETER, G. E. Mechanical metallurgy, 2 Ed., p. 204. Rio de Janeiro: McGraw-Hill Kogakusche Ltd. American Society for Metals-ASM, 1988.

10 EPE - Empresa de Pesquisa Energética. Projeção da demanda de energia elétrica para os próximos 10 anos. Rio de Janeiro: EPE, 2011. (Série Estudos de Energia). (Nota Técnica).

11 Grupo de Pesquisa em Engenharia de Materiais - GPEMAT. Disponível em: http://www.ufpa.br/gpemat/equipe.htm. Acesso em: 17 mai. 2017.

12 REED HILL, R. E. Princípios de Metalurgia Física, 2ª . Ed, Guanabara dois; Rio de Janeiro, 1982, p. 310. 